\title{
A Retrospective Study on the Adverse Effects of Chemotherapy in Breast Cancer-A Multicenter Study
}

\author{
Devender Kodati*, Sowmya Tirumala, Rishitha Krishna Kantheti, Joseph Priyatham Reddy Thumma
}

Department of Pharmacy Practice, St. Peter's Institute of Pharmaceutical Sciences, Warangal-506001, Telangana State, INDIA.

\begin{abstract}
Objective: The present study is a retrospective observational and a non-comparative descriptive study, to evaluate the clinical manifestations, risk factors, diagnosis, treatment regimen and mainly the adverse effects associated with the chemotherapeutic drugs, where the case sheets of the breast cancer patients were reviewed properly and the required data is obtained and finding of adverse effects was considered as the primary parameters. Methodology: A total of 353 patients were enrolled in the study. The required demographic details and laboratory data were reviewed from the case sheets. During chemotherapy, some of the patients were given with AC, $\mathrm{T}$ (Doxorubicin, cyclophosphamide, paclitaxel) regimen and few patients were given with CAF (Cyclophosphamide, Doxorubicin, 5-FU) regimen. The reports were re-reviewed for adverse effects after chemotherapy. Results: Among 353 patients, 344 patients have shown adverse effects and 9 patients were without any adverse effects during chemotherapy. We have identified 1,157 adverse effects in a total of 344 patients, among them $16.5 \%$ were found to be cardiac effects, $60.5 \%$ were found to be haematological effects and $22.6 \%$ were found to be in USG abdomen. Conclusion: Among 353 patients, age group between 49-58 years has shown higher adverse effects $(25.85 \%)$ and the least adverse effects were presented by the age group between $18-28$ years $(6.52 \%)$.
\end{abstract}

Key words: Breast cancer, Chemotherapy, Adverse effects, Doxorubicin, Cyclophosphamide, Paclitaxel.

\section{INTRODUCTION}

Cancer is a group or more than 100 different diseases that are characterized by uncontrolled cellular growth, local tissue invasion and distant metastases. ${ }^{1}$ Breast cancer is the most frequent cancer in female population. More than half of the cases occur in industrialized countries. ${ }^{2}$ Breast cancer has been top ranked among Indian females with age adjusted rate as high as 25.8 per 100,000 women and mortality 12.7 per 100,000 women. 1.67 million new breast cancer cases were diagnosed annually. Data reports from various latest national cancer registries were compared for mortality rates and incidence. The age adjusted incidence rate of carcinoma of the breast was found as high as 41 per 100,000 women for Delhi, followed by Chennai (37.9), Bangalore (34.4) and Thiruvananthapuram District (33.7). ${ }^{3}$ Certain risk factors exist for developing breast cancer are age, family history of any cancer, age of pregnancy, age of menstruation, entering menopause later (over age 55), oral contraceptives and hormone replacement therapy. ${ }^{4}$ The treatment plan for an individual patient depends on patient factors. Treatment in breast cancer include chemotherapy (AC, T / CAF regimens), surgery, radiation therapy, hormonal therapy and doses of the therapy depends on the patient physical condition, body weight, age and other co-morbidities. Chemotherapy commonly involves several severe adverse effects, but recent scientific advances mean that many of these are far more manageable than in the past. Adverse effects can range from mild to severe, depending on the type and extent of the treatment and other individual factors. Hence the scope of the study is to identify and manage the adverse effects by dose adjustment.

\section{MATERIALS AND METHODS}

Study site: The study was conducted in
DOI: 10.5530/ijopp.12.2.24

Address for

correspondence:

Dr. Devender Kodati, Associate Professor, Department of Pharmacy Practice, St. Peter's Institute of Pharmaceutical Sciences, Warangal-506001, Telangana State, INDIA.

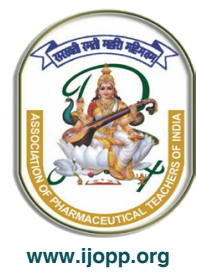


tertiary care Hospitals, Pharmacy Practice Department with a prime focus on variety of patient care processes with a basic objective of providing appropriate treatment to the disease.

Study design: A Retrospective observational study and a non-comparative descriptive study on the adverse effects of chemotherapy in breast cancer patients.

Study period: The study was conducted for a period of 6 months.

\section{Study criteria \\ Inclusion criteria}

Diagnosis: Breast cancer

Treatment plan: Chemotherapy regimen AC, $\mathrm{T}$ (Doxorubicin, cyclophosphamide, paclitaxel) and CAF (Cyclophosphamide, Doxorubicin, 5-FU).

Gender: Female

Age: Above 18 years

All patients with breast cancer irrespective of their co-morbid conditions.

\section{Exclusion criteria}

Patients less than 18 years of age

Critically ill patients

Gender: Males

Pregnant and lactating women

Patients receiving radiation therapy.

\section{Sample size}

A total number of 353 patients who received chemotherapy were included in the study.

\section{Treatment plan}

Study consists of different regimens of treatment that were given to patients at various stages of breast cancer, tumour size lymph node involvement and metastasis. The regimens of treatment applied were AC, T or CAF regimen.

\section{The regimen consists of different cycles}

AC, T regimen: In this regimen, AC is given for 4 cycles every 21 days followed by $\mathrm{T}$ for the next 4 cycles based on Body Surface Area (BSA).

CAF regimen: In this regimen CAF is given for 6 cycles every 21 days based on BSA.

\section{Procedure}

Data collection form was designed. Data was collected from patient's medication chart. Adverse effects like cardiological, haematological effects and the effects related to Ultrasound Sono Graphy (USG) abdomen were identified by reviewing the patient's case reports, laboratory reports before and after the treatment.

\section{Statistical analysis}

Statistics (confidence interval, chi-square test) were applied to characterize the whole study sample with regard to demographics, type of co-morbidities, type of treatment regimen and adverse effects of the chemotherapeutic drugs.

\section{RESULTS}

A study was conducted in a tertiary care hospital on 353 patients with histological diagnosis of breast cancer. Age wise distribution of prescriptions, prescription distribution based on the adverse effects, prescription distribution based on Immunohistochemistry, prescription distribution based on BMI, total number of adverse effects in the prescriptions, total number of adverse effects identified in each prescription and different adverse effects associated with the chemotherapy were identified.

In our study, out of 353 prescriptions, 9(2.5\%) prescriptions were without adverse effects and 344 $(97.5 \%)$ prescriptions were found with adverse effects (Table 1).

A total of 1,162 adverse effects were identified during chemotherapy. Of them 192 (16.5\%) were cardiological, $703(60.5 \%)$ were haematological and $262(22.6 \%)$ adverse effects were found in USG abdomen (Table 2).

The age wise distribution of prescriptions: $18-28$ years (7.08\%), 29-38 years $(18.71 \%), 39-48$ years $(26.06 \%)$, $49-58$ years $(24.36 \%), 59-68$ years $(16.15 \%), 69-78$ years (7.36\%) and $79-88$ years $(0.28 \%)$ were observed (Figure 1).

The highest percentage of adverse effects in age group of 49-58 years of age show $301(25.85 \%)$ adverse effects, $39-48$ years of age show $290(24.91 \%)$ adverse effects, 29-38 years of age show 196(16.83\%) followed by $59-68$ years of age show $189(16.23 \%)$ of adverse effects, $69-78$ years of age show $108(9.27 \%)$ of adverse effects, $18-28$ years of age show $76(6.52 \%)$ of adverse effects, $79-88$ years of age show $01(0.34 \%$ ) of adverse effects (Figure 2).

Indian Journal of Pharmacy Practice, Vol 12, Issue 2, Apr-Jun, 2019 


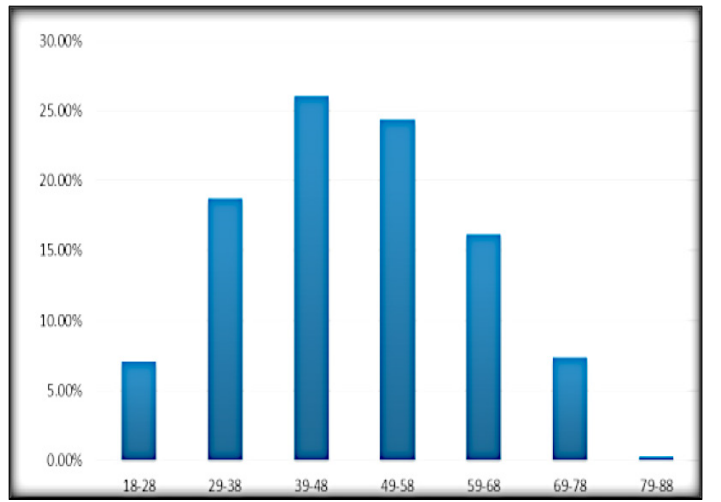

Figure 1: Age wise distribution of prescriptions in breast cancer patients receiving chemotherapy.

\begin{tabular}{|c|c|c|c|}
\hline $\begin{array}{l}\text { Presence of } \\
\text { adverse effects }\end{array}$ & $\begin{array}{c}\text { No. of } \\
\text { prescriptions }\end{array}$ & Percentage & $\begin{array}{c}\text { Confidence } \\
\text { interval }\end{array}$ \\
\hline $\begin{array}{c}\text { Without adverse } \\
\text { effects }\end{array}$ & 9 & $2.5 \%$ & \pm 1.63 \\
\hline $\begin{array}{l}\text { With adverse } \\
\text { effects }\end{array}$ & 344 & $97.5 \%$ & \pm 1.63 \\
\hline
\end{tabular}

Table 3: No. of adverse effects identified in each prescription of breast cancer patients receiving chemotherapy.

\begin{tabular}{cccc}
$\begin{array}{c}\text { No. adverse } \\
\text { effects }\end{array}$ & $\begin{array}{c}\text { No. of } \\
\text { prescriptions }\end{array}$ & Percentage & $\begin{array}{c}\text { Confidence } \\
\text { interval }\end{array}$ \\
\hline 0 & 09 & $2.5 \%$ & \pm 1.63 \\
1 & 37 & $10.4 \%$ & \pm 3.18 \\
2 & 66 & $18.6 \%$ & \pm 4.06 \\
3 & 84 & $23.7 \%$ & \pm 4.44 \\
4 & 80 & $22.6 \%$ & \pm 4.36 \\
5 & 46 & $13.03 \%$ & \pm 3.51 \\
6 & 22 & $6.2 \%$ & \pm 2.52 \\
7 & 08 & $2.2 \%$ & \pm 1.53 \\
8 & 01 & $0.2 \%$ & \pm 0.47 \\
\hline
\end{tabular}

Among 353 prescriptions $9(2.5 \%)$ prescriptions were without adverse effects of the rest 344 prescriptions 1157 adverse effects were identified. Among them 84 (23.7\%) prescriptions were reported with 3 adverse effects, 80 $(22.6 \%)$ prescriptions were reported with 4 adverse effects, $66(18.6 \%)$ prescriptions were reported with 2 adverse effects, 46 (13.03) prescriptions were reported with 5 adverse effects, $37(10.4 \%)$ prescriptions were reported with 1 adverse effect, $22(6.2 \%)$ prescriptions were identified with 6 adverse effects, $8(2,2 \%)$ prescriptions were identified with 7 adverse effects and $01(0.2 \%)$ prescriptions were identified with 8 adverse effects (Table 3).

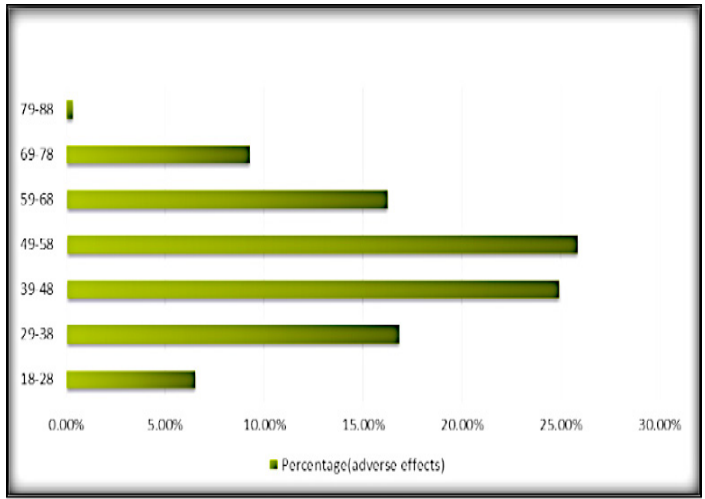

Figure 2: Age wise distribution of adverse effects in prescriptions among breast cancer patients receiving chemotherapy.

Table 2: Total number and different categories of adverse effects of chemotherapy in patients with breast cancer.

\begin{tabular}{cccc}
\hline Category & $\begin{array}{c}\text { No. of adverse } \\
\text { effects }\end{array}$ & Percentage & $\begin{array}{c}\text { Confidence } \\
\text { interval }\end{array}$ \\
\hline Cardiological & 192 & $16.5 \%$ & \pm 3.87 \\
Haematological & 703 & $60.5 \%$ & \pm 5.1 \\
USG Abdomen & 262 & $22.6 \%$ & \pm 4.36 \\
Total & 1157 & - & - \\
\hline
\end{tabular}

From our study, out of 353 prescriptions 230 prescriptions $(65.1 \%)$ were found to be normal, 53 prescriptions $(15.01 \%)$ were done with tubectomy, 47 prescriptions $(13.3 \%)$ were found to be hypertensive, 29 prescriptions $(8.21 \%)$ with hysterectomy, 19 prescriptions (5.3\%) were found to be Diabetic, 18 prescriptions $(5.09 \%)$ were done with LSCS and 5 prescriptions $(1.41 \%)$ of other past medical history (Table 4).

Out of 353 prescriptions 175 prescriptions (49.5\%) were right sided breast cancer and 178 prescriptions (50.5\%) were left sided breast cancer (Table 5).

Among 353 prescriptions, 157 prescriptions (44.48\%) were found to be menstrual and 196 prescriptions $(55.52 \%)$ were found to be menopausal (Figure 3).

Out of 353 prescriptions, 170 prescriptions (48.1\%) were of normal BMI, 72 prescriptions $(2.03 \%)$ were underweight, 78 prescriptions $(22.09 \%)$ were overweight, 33 prescriptions $(9.34 \%)$ were obese (Figure 4$).$

Among $99 \mathrm{CAF}$ regimen and $99 \mathrm{AC}$, T regimen 664 adverse effects were observed. CAF regimen shows 275(41.41\%) adverse effects whereas AC, T regimen shows 389(58.58\%) adverse effects (Table 6).

Out of 353 prescriptions 146 prescriptions (41.36\%) were found to be ER positive, 207 (58.6\%) prescriptions 


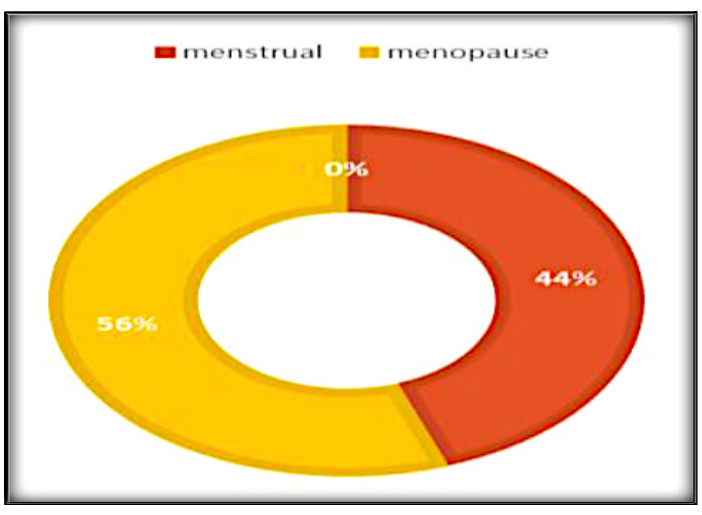

Figure 3: Prescription distribution based on menstrual status in breast cancer patients.

\begin{tabular}{cccc}
$\begin{array}{l}\text { Table 4: Prescription distribution with past medical } \\
\text { history (PMHx) in breast cancer patients receiving } \\
\text { chemotherapy. }\end{array}$ & $\begin{array}{c}\text { No. of } \\
\text { prescriptions }\end{array}$ & Percentage & $\begin{array}{c}\text { Confidence } \\
\text { interval }\end{array}$ \\
\hline PMHx & 18 & $5.09 \%$ & \pm 2.29 \\
\hline LSCS & 29 & $8.21 \%$ & \pm 2.86 \\
Hysterectomy & 53 & $15.01 \%$ & \pm 3.73 \\
Tubectomy & 19 & $5.3 \%$ & \pm 2.34 \\
Diabetes & & $13.3 \%$ & \pm 3.54 \\
mellitus & 47 & $1.41 \%$ & \pm 1.23 \\
Hypertension & 5 & $65.1 \%$ & \pm 4.97 \\
Others & 230 & & \\
Normal & &
\end{tabular}

Table 5: Prescription distribution based on morbidity of left and right-side breast cancer.

\begin{tabular}{cccc} 
Morbidity & $\begin{array}{c}\text { No. of } \\
\text { prescriptions }\end{array}$ & Percentage & $\begin{array}{c}\text { Confidence } \\
\text { interval }\end{array}$ \\
\hline $\begin{array}{c}\text { Right sided } \\
\text { BC }\end{array}$ & 175 & $49.5 \%$ & \pm 5.22 \\
$\begin{array}{c}\text { Left sided } \\
\text { BC }\end{array}$ & 178 & $50.5 \%$ & \pm 5.22 \\
\hline
\end{tabular}

\begin{tabular}{|c|c|c|c|}
\hline Regimen & $\begin{array}{l}\text { No. of Adverse } \\
\text { effects }\end{array}$ & Percentage & $\begin{array}{c}\text { Confidence } \\
\text { interval }\end{array}$ \\
\hline CAF & 275 & $41.41 \%$ & \pm 9.7 \\
\hline $\mathrm{AC}, \mathrm{T}$ & 389 & $58.58 \%$ & \pm 9.7 \\
\hline
\end{tabular}

were found to be ER negative, 186 prescriptions (52.7\%) were found to be PR positive, 167 prescriptions (47.39\%) were found to be PR negative, 86 prescriptions $(24.36 \%)$ were found to be ER-PR positive and 65 prescriptions (18.41\%) were found to be ER-PR negative.

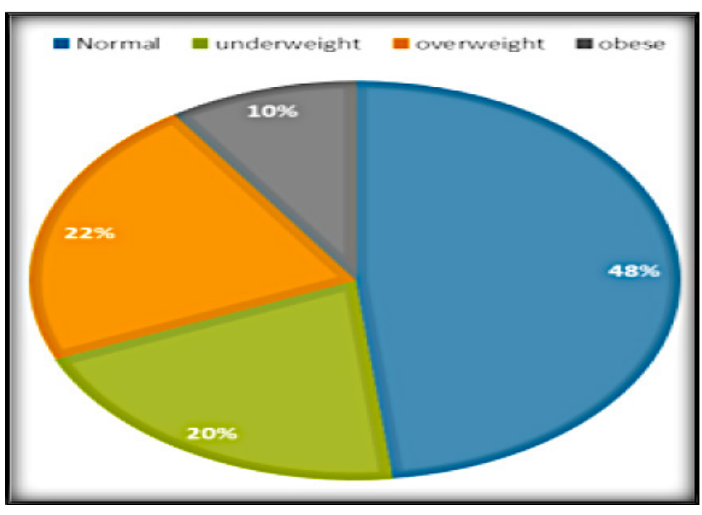

Figure 4: Prescription distribution based on BMI in breast cancer patients.

\section{DISCUSSION}

A study was conducted in a cancer hospital of Oncology Department to evaluate the adverse effects of chemotherapy in breast cancer. Age wise distribution, symptoms associated with breast cancer, treatment regimen and the outcomes, the adverse effects associated with the treatment regimen were being evaluated.

In the present study, it was observed that predominant number of patients visited the hospital were diagnosed with breast cancer.

A total number of 353 prescriptions were reviewed from in-patient case records in a tertiary care hospital during a period of 6 months. Of them 344 (97.5\%) prescriptions have reported 1,157 adverse effects and 9 $(2.5 \%)$ prescriptions were without adverse effects.

A study conducted by John J. Doyle et al. ${ }^{5}$ showed that chemotherapy especially with anthracyclines was associated with a three-fold increase in the incidence and severity of anthracycline - induced cardiotoxicity seems to persist over time. Among 31,748 women in the study, 5,575 received chemotherapy of which $22 \%$ had increased risk of cardio toxicity. In the present study $16.5 \%$ of the patients were developed with cardiac effects which are similar to the above study.

A study conducted by Michael J. Hassett et al. ${ }^{6}$ shows that the association between chemotherapy related serious adverse effects and chemotherapy administration was assessed using odds ratio. In the present study chemotherapy related adverse effects like left ventricular diastolic dysfunction, tachycardia, alopecia, darkening of nails, dizziness, headache, haematological effects like anaemia, neutropenia and also increased serum creatinine levels, changes in diagnosis of USG abdomen were observed. Women who received chemotherapy had statistically significantly greater odds of experiencing each Indian Journal of Pharmacy Practice, Vol 12, Issue 2, Apr-Jun, 2019 
of eight chemotherapy-related serious adverse effects and 3.6-fold ( $95 \% \mathrm{CI}=3.0$ to 4.3 ) greater odds of experiencing at least one of the eight chemotherapy related serious adverse effects.

A study conducted by Julie Lemieux et al. ${ }^{7}$ shows chemotherapy-induced alopecia and effects on quality of life among women with breast cancer. The major adverse effect of chemotherapy is alopecia. This is the common side effect of chemotherapies used in the treatment especially in case of breast cancer. In the present study, most of patients experienced alopecia which is similar to the above study

In the present study, levels of serum creatinine were increased in most of the patients which indicates renal toxicity and most of patients experienced different grades of left ventricular diastolic dysfunction during chemotherapy. This is similar to the study conducted by Hurria A et al. ${ }^{8}$ The study conducted by Hurria A et al. shows that out of 132 patients with primary invasive breast cancer who received one of three different chemotherapy protocols: cyclophosphamide, methotrexate, 5-fluorouracil (CMF), doxorubicin and cyclophosphamide (AC) or AC plus paclitaxel or docetaxel (AT-T). Mean age was 70 years and co-morbidity was low as measured by the Charlson Co-morbidity Index. $5 \%$ had a score of $2,12 \%$ had a score of $1,83 \%$ of patients had a score of 0 co-morbidities. Patients who received anthracycline based regimens were more likely to experience grade 3 or 4 toxicity $(P=0.01)$.

Cutaneous complications are majorly observed in the breast cancer patients, where darkening of nails along with finger tips and also darkening of veins was observed. This was the complication of cancer chemotherapy. This is similar to the study conducted by Dreizen et al. ${ }^{9}$ which shows that the agents used in cancer chemotherapy are highly potent and have only a slight margin of safety between toxic and the tumoricidal doses. The skin is a most frequent target for the side effects of these drugs. Major cutaneous manifestations of toxicity include alopecia, cellulites, hyper pigmentation, haemorrhage and infection.

In the present study, patients were observed with grade I, II, III, IV left ventricular diastolic dysfunction. This was the adverse effect of chemotherapy during treatment which was identified through 2D Echo diagnostic test. This is similar to the study conducted by Daniela Cardinale et al..$^{10}$ in which the left ventricular dysfunction was predicted early by Troponin I release after high dose chemotherapy where in the present study left ventricular dysfunction was observed by $2 \mathrm{D}$ echo cardiogram.
During the chemotherapy of breast cancer many adverse effects were identified in the present study. Among them hematological adverse effects accounts for about $60.5 \%$ of total number of adverse effects. Abnormal levels of the blood cells were identified. There was a deviation from the normal ranges of the blood cells. This study is similar to the study conducted by Shahrasbi A et al. ${ }^{11}$ which concluded that Cytopenia was more prevalent. In the present study, out of 353 Patients, 252 patients were diagnosed with anaemia. In the present study, among 353 prescriptions 99 were found to be CAF regimen with a total of 275 adverse effects and 254 prescriptions were found to be of AC, T regimen. From 254 prescriptions 99 prescriptions were randomly picked and among them 378 adverse effects were observed. From the above discussion CAF regimen has less adverse effects.

\section{CONCLUSION}

In the present study, 353 female patient prescriptions were recruited in tertiary care hospitals. Of 353 prescriptions, 344 prescriptions were found with adverse effects i.e. around $97.5 \%$ of patients showing adverse effects for chemotherapy in breast cancer. Total adverse effects were found to be 1,157 from 344 prescriptions. According to the study 49-58 years of the patients show high adverse effects compared to the other age groups. Based on the diagnostic data the patients show $192(16.5 \%)$ of cardiological effects, $703(60.5 \%)$ of haematological effects, $262(22.6 \%)$ of USG abdomen effects. From the study minimum adverse effects is one and highest number of adverse effects is eight while others (only 9 prescriptions) show no adverse effects. From the data it was observed that most number of prescriptions show 3-4 adverse effects. Among 353 patients 157 patients are on their regular menstrual cycle where as other 196 patients are menopausal. The most common adverse effects are alopecia, darkening of nail and finger tips, veins, anemia, neutropenia, left ventricular diastolic dysfunction and increased serum creatinine. From this observational study the more number of adverse effects (58.58\%) were observed in AC, T regimen compared to CAF regimen (41.41\%) adverse effects.

\section{ACKNOWLEDGEMENT}

Authors would like to thank the Hospital Management of Susrutha Cancer hospital, Karimnagar, Telangana and St. Ann's Cancer hospital, Warangal, Telangana and the patients for their cooperation. 


\section{CONFLICT OF INTEREST}

The authors declare no conflict of interest.

\section{ABBREVIATIONS}

AC, T: Doxorubicin, Cyclophosphamide, Paclitaxel; CAF: Cyclophosphamide, Doxorubicin, 5-FU; 5-FU: 5-Flurouracil; USG: Ultrasound Sonography; BSA: Body Surface Area; BMI: Body Mass Index; PMHx: Past Medical History; LSCS: Lower Segment Caesarean Section; IHC: Immunohistochemistry; ER-PR: Estrogen Receptor-Progesterone Receptor.

\section{SUMMARY}

A retrospective study was performed in breast cancer patients receiving chemotherapy to find out the adverse effects. A total of 353 patients were included in the study, out of which $97.5 \%$ of patients have shown adverse effects and $2.5 \%$ of patients were without any adverse effects. A total of 1,157 adverse effects were identified in 353 prescriptions. Among them 16.5\% cardiological, $60.5 \%$ haematological and $22.6 \%$ in USG abdomen were identified. Some of the common adverse effects like alopecia and darkening of nails and finger tips were directly observed in the patients.

\section{REFERENCES}

1. Bruce AC, Dan L. Cancer chemotherapy and Biotherpy. Sultan Qaboos University Medical Journal. 2011;11(3):424-5.

2. Parkin DM, Bray F, Ferlay J, Pisani P. Global cancer statistics. CA Cancer J Clin. 2005;55(2):74-108.

3. Malvia S, Bagadi SA, Dubey US, et al. Epidemiology of breast cancer in Indian women. Asia Pacific Journal of Clinical Oncology. 2017;13(4):289-95.

4. Prentice RL, Sheppard L. Dietary fat and Cancer: Consistency of the epidemiologic data and disease prevention that may follow from a practical reduction in fat consumption. Cancer Causes Control. 1990;1(1):81-97.

5. John JD, Alfred IN, Judith SJ, et al. Chemotherapy and Carditoxicity in Older Breast Cancer Patients: A Population-Based Study. Journal of Clincical Oncology. 2005;23(34):8597-605.

6. Hassett MJ, O'Malley AJ, Pakes JR, et al. Frequency and Cost of ChemotherapyRelated Serious Adverse Effects in a Population Sample of Women with Breast Cancer. Journal of the National Cancer Institute. 2006;98(16):1108-17.

7. Julie L, Elizabeth M, Lousie P. Chemotherapy-induced alopecia and effects on quality of life among women with breast cancer: A literature review. Psychooncology. 2008;17(4):317-28.

8. Hurria A, Brogan K, Panageas KS, et al. Patterns of toxicity in older patients with breast cancer receiving adjuvant chemotherapy. Breast Cancer Res Treat. 2005;92(2):151-6.

9. Dreizen S, Bodey GP, Rodriquez V, et al. Cutaneous complications of cancer chemotherapy. Postgrad Medicine. 2016;58(6):150-8.

10. Daniela C, Maria TS, Alessandro M, et al. Left Ventricular Dysfunction Predicted by Early Troponin I Release After High-Dose Chemotherapy. Journal of the American College of Cardiology. 2000;36(2):517-22.

11. Shahrasbi A, Armin A, Ardebili A, et al. Hematologic Adverse Effects following Systemic Chemotherapy. Journal of Oncology Medicine and Practice. 2017;2(1):110. 